\title{
Shape of U: The relationship between object-location memory and expectedness
}

\author{
Jörn Alexander Quent*1, Andrea Greve ${ }^{1}$, \& Richard Henson ${ }^{1,2}$ \\ ${ }^{1}$ MRC Cognition \& Brain Sciences Unit, University of Cambridge, UK \\ ${ }^{2}$ Department of Psychiatry, University of Cambridge, UK
}

*Corresponding author:

Jörn Alexander Quent

MRC Cognition \& Brain Sciences Unit

University of Cambridge

15 Chaucer Road

Cambridge, CB2 7EF

UNITED KINGDOM

Telephone: +44 1223355294

Email: alex.quent@mrc-cbu.cam.ac.uk

RUNNING TITLE: SHAPE OF U

Acknowledgements: This work was supported by the UK Medical Research Council.

(SUAG/046 G101400). JAQ's PhD studentship is funded by the Gates Cambridge Trust. 


\section{Abstract}

Based on a neuroscientific model of memory (SLIMM), we predicted that people's memory for object locations would be a U-shaped function of the expectancy of those locations. Using immersive virtual reality, we manipulated expectancy by placing twenty familiar objects in locations within a virtual kitchen that were congruent, unrelated or incongruent with people's schema (prior knowledge) for a typical kitchen. Using Bayes Factors across three experiments, we confirmed this (pre-registered) prediction, with better memory for highly expected or highly unexpected locations relative to neutral locations. This U-shape was found in location recall and, importantly, in three-alternative forced choice recognition using object-location images, for which locations in the foil images were approximately equally expected. The latter shows that (part of) the U-shape was not simply participants guessing expected locations when unsure. A second prediction of SLIMM is that the two ends of the U-shape would be associated with different expressions of memory: namely, recollection of objects at unexpected locations, but familiarity for objects at expected locations. Bayes Factors provided evidence against this second prediction, with recollection associated with both ends of the U-shape, and familiarity showing no effect of expectancy. These findings have implications for SLIMM and more general theories of the role of schema and surprise in episodic memory.

Keywords: prediction error, schema, virtual reality, memory 


\section{Introduction}

Key to everyday functioning is the ability to predict aspects of our environment, and one source of such predictions is a "schema" (Bartlett, 1932), i.e., abstracted knowledge about recurring situations, such as what to expect when walking into a kitchen (compared to a bathroom, or other type of room). Such schemas also influence how we encode new experiences into memory (Bransford \& Johnson, 1972), which in turn have the potential to update or form new schemas in future.

Numerous studies have shown that memory is better for information that fits with our prior knowledge, i.e., is expected from a schema (Alba \& Hasher, 1983; Anderson, 1981; Craik \& Tulving, 1975). This so-called "congruency effect" has been obtained using a broad range of memoranda (e.g., Bein et al., 2015; Brod \& Shing, 2019; van Buuren et al., 2014). However, many other studies show the apparent opposite finding, of better memory for unexpected or surprising information, i.e. that is incongruent with a schema (Brod et al., 2018; Greve et al., 2017; Hunt \& Worthen, 2006; von Restorff, 1933).

This raises the question how information that is congruent or incongruent with a schema can both be remembered well. A recent neuroscientific model called SLIMM (Schema-Linked Interactions between Medial Prefrontal and Medial Temporal Lobe; van Kesteren et al., 2012) proposes that memory is a Ushaped function of schema-congruency, i.e., the degree of expectancy of encountering a specific event, given a schema. More specifically, it is hypothesized that different brain systems support memory for the two ends of the U-shape: one based in the medial temporal lobes (MTL), including the hippocampus, which best encodes highly unexpected (schema-incongruent) events, and another involving the medial prefrontal cortex (MPFC), which best encodes highly expected (schemacongruent) events. We previously found support for this U-shaped prediction using a paradigm in which participants first learned a new schema (a rule about the relative value of two types of object; Greve et al., 2019). However, this paradigm only enabled three levels of expectancy/congruency, and the conclusions were contingent on the "middle" condition (what we called the "unrelated" condition) 
being equivalent to the two extreme conditions (the "congruent" and "incongruent" conditions) in all ways other than congruency with the learned rule. Furthermore, while abstract, the learned rule was arguably a trivial case of the much richer schema that operate in everyday life, and which have typically been learned over many years prior to participating in a laboratory experiment.

Our first aim in the present study was therefore to replicate this U-shaped function of memory for new information as a more continuous function of schema-congruence (or what we will call "expectancy"), using a much more realistic, pre-experimental schema. To this end, we used immersive virtual reality (iVR) to "place" participants in a life-like situation (a virtual kitchen), and tested their memory for the location of objects as a function of how likely those objects were to appear in that location (i.e., based on their pre-existing schema for typical locations to find objects in a kitchen). So for example, a kettle might appear on the kitchen counter (expected), on a kitchen table (neither strong expected nor unexpected) or on top of a trashcan (unexpected). We then tested participants' memory using both recall, where they had to place a given object at the location where they remembered it, and recognition, where they had to select which of the three images of an object in different locations corresponded to the location they remembered (i.e., three-alternative forced choice, 3AFC).

One possible explanation for the $\mathrm{U}$-shape is that unexpected locations are surprising, attract attention and this improves memory encoding. Expected locations, however, are not necessarily encoded better, but simply benefit at retrieval owing to the tendency for participants to guess an expected location (based on their schema) when they cannot remember the specific location. To control for the latter guessing effect, we ensured that the two foils used in the $3 \mathrm{AFC}$ test showed locations for the target object that were approximately equally expected (based on normative ratings). If the U-shape remains in 3 AFC performance, then this suggests that the memory advantage for expected locations also occurs at encoding, supporting the idea of two separate memory systems. 
A second prediction of the SLIMM model follows from its assumption that the two memory systems operate according to different mechanisms, evolved for different goals. It makes sense for the MTL system that underlies the memory advantage for surprising events (those that produce a high "prediction error") to encode an episodic "snapshot" of such events, which includes surrounding contextual information that appears incidental to the prediction (schema). Retaining such incidental information is likely advantageous (if it keeps recurring) for updating the schema so that the same prediction error does not occur in future. For example, if one is surprised to find boiling water coming from a tap on a kitchen sink, one should remember details about that tap, and update one's kitchen schema so that, when encountering such taps again, one knows that making tea is done using boiling water from these taps (rather than from a kettle). Therefore the SLIMM model predicts that memory of unexpected information will be accompanied by recollection of its episodic context.

On the other hand, the mPFC system underlying memory for expected information is assumed to enable rapid integration of new information into an existing schema, during which incidental (contextual) information is lost, because it is not part of that schema. So if a kettle that you use for the first time in someone's kitchen behaves exactly as expected, you are unlikely to encode details about that kettle, such as where it was stored. SLIMM therefore predicts that memory for expected information will be associated with a feeling of familiarity instead, in the absence of recollection of the original context. We therefore used a variant of the "remember/know" procedure (Tulving, 1985) to assess whether memory for unexpected locations was more likely to be associated with "remember" responses while memory for expected locations was more likely to be associated with "familiar" responses.

In summary, we used iVR to test people's memory for the location of everyday objects within a virtual kitchen. The expectancy of each object location was derived from normative ratings of 20 objects in each of 20 possible locations, from which locations were selected to cover a range from highly expected $(+100)$ to highly unexpected $(-100)$. Participants spent 45 seconds counting these 20 objects 
in the virtual kitchen, before the objects were removed for a short delay, and participants were asked to replace individual objects at their remembered location, followed by the final 3AFC test outside iVR (using stills on a computer screen). Our two main predictions were: 1 ) recall and recognition for object location would be a U-shaped function of expectancy of an object's location, as tested by a quadratic component and interrupted linear regression, and 2) recollection, as estimated from remember/familiar judgments, would decline with expectancy, while familiarity would increase with expectancy. To allow for individual differences in schema, expectancy ratings were defined individually, based on a debriefing phase.

We tested these two hypothesis across three experiments. The first experiment was an initial attempt to obtain a basic U-shape, while the next two added a measure of recollection/familiarity to additionally test the second hypothesis. Apart from the first experiment, the experiments were preregistered, and we use Bayesian analysis to propagate evidence across experiments, i.e., using the posterior of one experiment as the prior for the next experiment (similar conclusions are reached when using pooled frequentist analysis; see Supplementary Material Table S.6.5).

\section{Experiment 1}

Experiment 1 aimed to detect a basic U-shape for object-location memory as a function of expectancy of that location, and estimate its effect size. This was defined by the quadratic coefficient of a secondorder polynomial expansion of individuals' continuous expectancy ratings.

\section{Methods}

\section{Participants}

All participants in the present study were Cambridge community members from the volunteer panel of the MRC Cognition and Brain Science Unit, all of whom had reported normal or corrected-to-normal visual acuity, provided informed consent and received monetary compensation for participation, as 
approved by a local ethics committee (CPREC 2020.018). Each volunteer only participated in one of the present experiments.

Because Experiment 1 was the first experiment on this paradigm, no effect size was available to power the study, and so we decided to test 16 participants, as a number typical of laboratory memory experiments. These participants had a mean age of $26.38(S D=3.52)$, with eight females. For one participant, information was lost about whether one object was seen at study. However, recordings of their object placement was intact, so we kept this participant, given that it was rare for objects to be reported as not seen.

\section{Materials}

Stimuli comprised 12 kitchen objects and 8 non-kitchen objects (Supplementary Table S1), inspired by Lew \& Howe (2017). A normative study was run to obtain 400 expectancy ratings for each of the 20 objects at each of 20 possible locations in the kitchen (Supplementary Material S2). From these, a single assignment of each object to one of 20 locations was chosen so as to maximise the range of expectancies (Supplementary Material S3.1). The virtual kitchen was 5.15 by 4.40 virtual-meter (vm) kitchen, where $1 \mathrm{vm}$ corresponds to approximately $1 \mathrm{~m}$ in the real-world (material available here https://osf.io/4sw2t/).

\section{Procedure}

The basic paradigm for all four experiments is illustrated in Figure 1, and contains an encoding and recall phase performed in iVR, followed by a recognition and expectancy-rating phase performed on a computer screen. During encoding, participants were asked to navigate freely through a virtual kitchen for $45 \mathrm{sec}$, with the instruction to count and memorise the locations of all 20 objects that were scattered across the room. Following encoding and prior to recall, participants entered a blank room for approximately $2.5 \mathrm{~min}$ to practice how to place new objects (simple cubes) using the iVR hand controls; a skill that was needed in the subsequent recall phase. For this recall, participants re-entered 
the kitchen (now without the 20 original objects), were given one object, and asked to place it at its previously seen location (once placed, the object disappeared, and the process was repeated for the remaining 19 objects). Participants were encouraged to guess if they were unsure, but could skip if they did not remember the object at all (a miss). Recall accuracy was defined as whether or not the correct location was the closest of the 20 object locations to the recalled location (equivalent results were found when using a more continuous Euclidean metric of distance from the correct location, as shown in Supplementary Material Table S.6.4).

I) Encoding

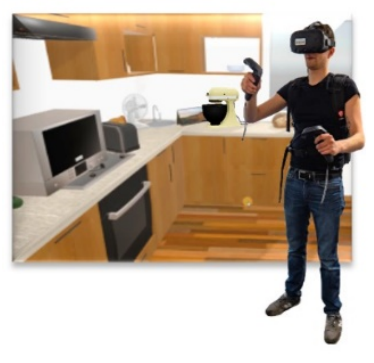

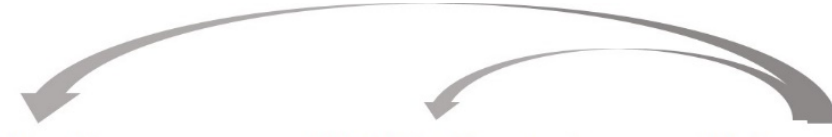

II) Recall

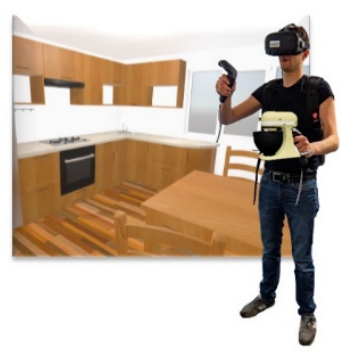

III) 3AFC (location)

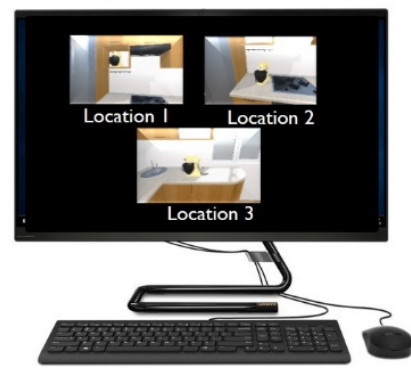

IV) Expectancy rating

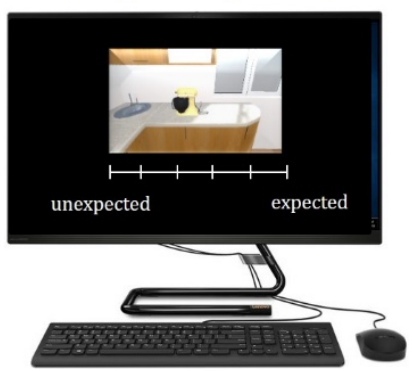

Figure 1. Schematic overview of paradigm, which differed across the three experiments only in object-location pairings and precise memory tests. I) Encoding (in iVR): participants explored a virtual kitchen (45 sec) and instructed to count and memorise 20 object locations. II) Recall (in iVR): all 20 objects were removed, and participants were given one object one at a time to be placed where previously encoded. III) 3AFC (outside iVR): each of the 20 objects was presented on a computer screen but in three alternative locations (where the two foil locations were approximately equally expected as the correct location). Experiments 2 and 3 collected additional remember/familiar judgments. IV) Individual expectancy ratings (outside iVR), used in analysis of previous Recall and 3AFC data, were collected for all 20 objects for a) each of the 3AFC locations and b) their general expectancy in a kitchen context.

Recall was followed by a $3 A F C$ recognition test, performed on a computer outside iVR. Each trial showed one studied object in three alternative locations, one of which was correct. Importantly, the two foil locations were chosen to be approximately equally expected according to the normative ratings (see above), so using prior knowledge to guess the location should not help performance. Participants indicated which they thought was the studied location, followed by a rating of their 
confidence on a 3-point scale ( $1=$ did not see the object, $2=$ guess the object was there, $3=$ know the object was there). Preliminary analysis of confidence did not add any new information (Supplementary Table 56.1), so here correct responses were defined as those given either a 2 or a 3 . In the final phase, participants provided expectancy ratings for how likely they thought it would be to find each of the 20 objects in each of the three locations tested in $3 \mathrm{AFC}$, together with an additional rating of the general expectancy of an object appearing anywhere in a kitchen at all. Ratings were made using a sliding scale from unexpected $(-100)$ to expected $(+100)$. These ratings were analogous to the normative ratings, but allowed for potential individual differences in expectancy.

\section{Statistical analysis}

Statistical analysis was performed in R (R Core Team, 2019) using Bayesian multi-level models with brms (Bürkner, 2017, 2018; version 2.12.0) based on Stan (Carpenter et al., 2017). All analyses scripts and data are available here: https://osf.io/4sw2t/

Memory for individual trials was modelled as a function of a participant's object-location expectancy rating. Memory was a binary outcome (correct/incorrect) and was fitted using logistic regression models with the Bernoulli linking function. A "full" model was fit first, with random slopes and intercepts for both objects and participants. Bayes Factors (BFs) using marginal likelihoods from bridge sampling (see Gronau et al., 2017) were then used to compare the full model with the model with random intercepts only, which was compared to the model without random intercepts.

The individually-defined expectancy ratings (Supplementary Figure S4) were scaled to have a standard deviation (SD) of 0.5 , and the prior for each regression coefficient was based on a "Student $t^{\text {" }}$ distribution, with hyperparameters of $\mathrm{df}=7, \mu=0, \sigma=1$, except for the intercept, which had hyperparameters of $\mathrm{df}=7, \mu=0, \sigma=10$ (see https://jaquent.github.io/post/the-priors-that-i-use-forlogsitic-regression-now/ for justification). These generic weakly informative priors are chosen to regularise unexpectedly large effects (Gelman et al., 2008). Eight Markov chain Monte Carlo (MCMC) 
chains were run, with 2000 warm-up and 16000 regular iterations and a total of 112,000 post-warmup samples for each main model. All models converged with an $\hat{R}$ of 1 .

Evidence for or against our hypotheses were quantified by BFs for the linear and quadratic component of a second-order polynomial expansion of expectancy. A symmetrical U-shape would have a positive quadratic coefficient and a zero linear coefficient (see Experiment 3 for a more stringent test based on opposite signs of interrupted linear regression). The BF for each coefficient was estimated by the Savage-Dickey ratio (Wagenmakers et al., 2010). The test for the quadratic term was order-restricted (one-tailed) in line with our pre-registered hypothesis, all other tests were not order-restricted unless otherwise specified. In short for one-tailed tests, we compared the density of the truncated and renormalised prior distributions at zero with the logspline non-parametric density estimate of the truncated and renormalised posterior distributions of our parameters at zero (based on the 112,000 post-warm-up samples). For unrestricted comparisons, BFs were just density ratios at zero: of prior/posterior $\left(B F_{10}\right)$ or posterior/prior $\left(B F_{01}\right)$ without truncation and renormalisation. The SavageDickey ratio function used can be found in this GitHub repository: https://github.com/JAQuent/assortedRFunction. In addition to BFs, we report $95 \%$ credible intervals around our parameters, which can be interpreted as evidence against the null hypotheses if they do not include zero.

Finally, when testing the means across trials, BFs were derived from Bayesian t-tests (Morey \& Rouder, 2018) with the package 'Bayes factor' (version 0.9.12-4.2), with the default scale parameter of $\sqrt{2} 2 / 2$.

\section{Results}

Objects that were reported as 'not seen' were excluded from further analysis. Across both the recall and 3AFC task, a mean of $2.84(S D=1.96)$ objects were excluded.

For the recall data (Figure 2A), a model with random intercepts for participants and objects was used, since this was favoured relative to one that also included random slopes $(B F=1018.85)$ and relative 
to one that did not have random intercepts $(B F=1593.28)$. There was little evidence for a linear effect, $\beta=0.096(95 \% \mathrm{Cl}[-0.663,0.885]), \mathrm{BF}_{10}=0.39$, but more importantly, there was strong evidence for the predicted positive quadratic effect, $\beta=1.888(95 \% \mathrm{Cl}[0.315,3.641]), \mathrm{BF}_{10}=25.04$.

As a control analysis, we added general expectancy (e.g. "how expected is this mixer in general in the kitchen?") as a linear covariate to the model. However, there was no evidence that this had any additional effect, $\beta=-0.747(95 \% \mathrm{Cl}[-1.61,0.169]), \mathrm{BF}_{10}=1.73$. Moreover, the estimated linear effect of location expectancy, $\beta=0.112$ (95\% $\mathrm{Cl}[-0.618,0.889]), \mathrm{BF}_{10}=0.38$, as well as the quadratic effect, $\beta$ $=2.092(95 \% \mathrm{Cl}[0.468,3.907]), \mathrm{BF}_{10}=40.91$, remained largely unchanged .
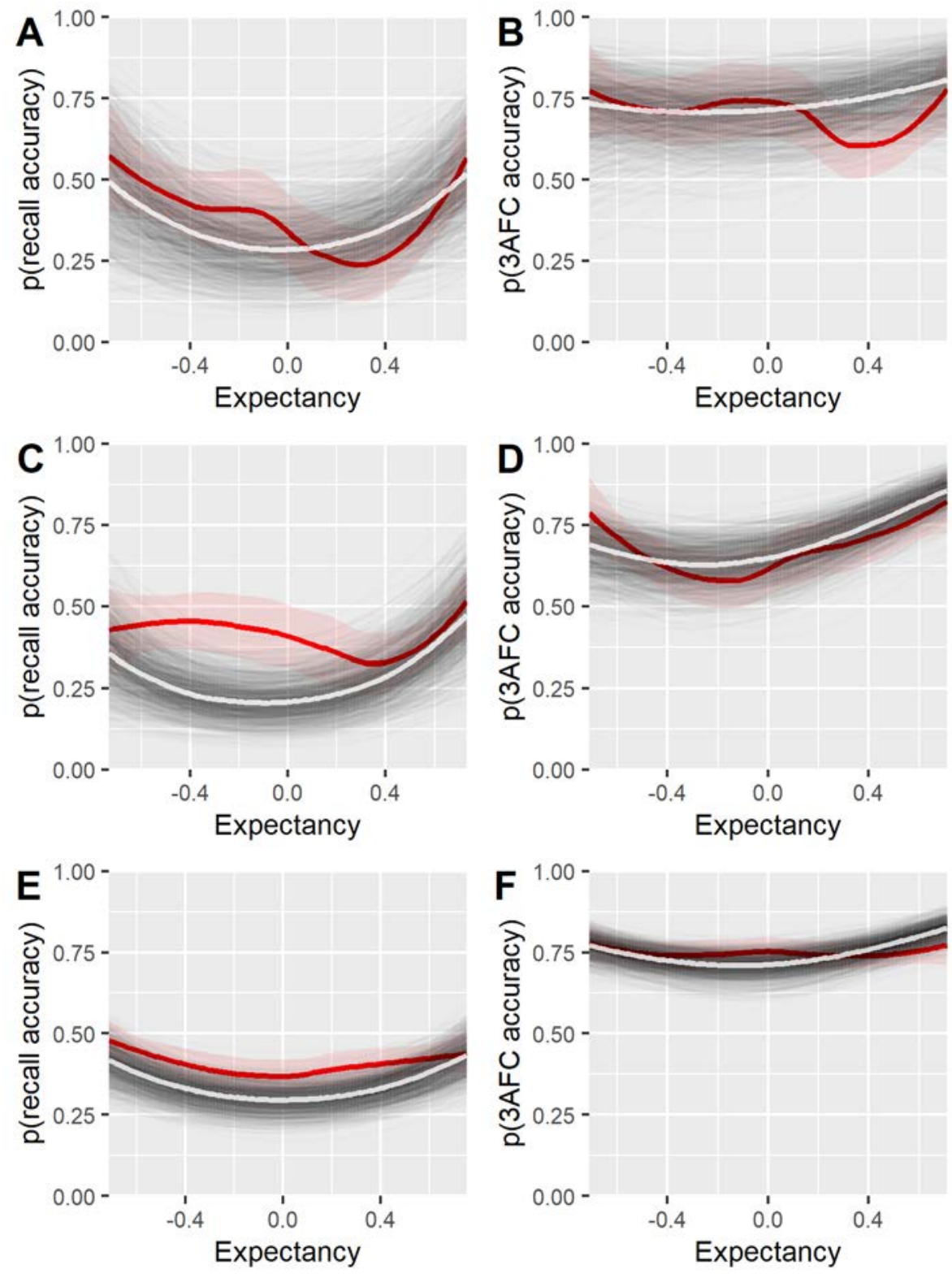
Figure 2. Recall (left panels) and recognition (right panels) performance plotted across expectancy ratings for all three experiments (rows). The red line shows locally weighted smoothing (loess) of data to illustrate how well the fitted $2^{\text {nd }}$-order polynomial model (white line) represents the data. The thin grey lines show 1,000 randomly selected fits from the posterior distributions, illustrating the uncertainty of the fit. Expectancy ratings originally ranged from -100 to +100 , but were scaled to have $S D=0.5$ in order to enable standard priors.

It is possible that the two sides of the U-shape arise at different stages, e.g., an advantage in encoding unexpected locations and an advantage in retrieving expected locations. To test the latter - i.e., whether expectancy influenced retrieval (e.g. guessing) - we compared the mean expectancy of incorrectly versus correctly recalled locations. Confirming expectations, the mean expectancy of incorrectly recalled locations was +40.7 (14.38), which was greater than zero, and greater than that for correctly recalled locations of $+2.9(10.6), \mathrm{BF}_{10}=23914, \mathrm{~d}=2.19$, supporting a bias for participants to report expected locations when unsure.

The 3AFC foils were designed to control for this potential bias towards recalling expected locations. Similar to recall, model comparison showed that the 3AFC data were better fit by a model with random intercepts but not random slopes $(\mathrm{BF}=717.10)$ and compared to a model with no random intercepts $(B F=19.45)$. This model again showed no evidence for or against a linear effect, $\beta=0.29$ (95 \% Cl $[-$ $0.48,1.12]), \mathrm{BF}_{10}=0.49$. However, in this case, the evidence for a quadratic effect was also unclear, $\beta$ $=0.64(95 \% \mathrm{Cl}[-0.72,2.07]), B F_{10}=1.61$ (Figure 2B). There was evidence against a linear effect of general expectancy when it was added as a covariate, $\beta=-0.27(95 \% \mathrm{Cl}[-1.15,0.68]), \mathrm{BF}_{01}=1.82$, and this addition had little effect on the linear, $\beta=0.29(95 \% \mathrm{Cl}[-0.48,1.11]), \mathrm{BF}_{10}=0.48$, or quadratic component, $\beta=0.697$ (95\% Cl $[-0.689,2.173]), \mathrm{BF}_{10}=1.79$, of expectancy ratings.

\section{Discussion}

Experiment 1 confirmed the predicted U-shape function for recall of object locations as a function of the expectancy of those locations. However, this U-shape was not replicated when using a 3AFC recognition test in which the foils were matched for expectancy, suggesting that some aspects of the U-shape might arise during retrieval rather than encoding, such as the bias towards guessing expected 
locations that was also observed. Nonetheless, the evidence from the $3 \mathrm{AFC}$ data was moot, and more data might be required to provide more compelling evidence.

Furthermore, a potential confound in Experiment 1 was that kitchen objects tended to be in highly congruent/incongruent locations, while non-kitchen objects primarily occupied by neutral locations. Even though there was evidence against any effect of general expectancy (of finding an object in the kitchen), and the inclusion of this effect in the model had negligible effect on the polynomial fit by individual object-location expectancies, we addressed this in the next experiment.

\section{Experiment 2}

Experiment 2 was powered to have a better chance of detecting the predicted U-shape in 3AFC performance. The procedure was identical to Experiment 1, except that the specific object-locations studied were re-selected (from the normative ratings) to give a more even coverage across the range of expectancy values for both kitchen and non-kitchen objects.

The only other important difference is that participants were also asked to indicate the quality of their memory responses by using 'remember/familiar/guess' judgements (Gardiner et al., 2002; Rajaram, 1993). This was to test the second hypothesis of SLIMM, that the two ends of the U-shape would be associated with different types of memory, specifically recollection for unexpected locations and familiarity for expected locations. This experiment was pre-registered on OSF (https://osf.io/s9er3).

\section{Methods}

\section{Participants}

We aimed for 24 participants based on the effect size in Experiment 1. These had a mean age of 25.00 $(S D=2.89)$ years, with 17 female, 6 male and 1 non-binary. Sample size was determined based on frequentist power analysis to achieve $80 \%$ power (https://osf.io/gr98d/) based on the quadratic effect size for 3AFC (https://osf.io/s9er3).. 


\section{Materials}

The only change is that objects were now reshuffled to other locations, so that kitchen and nonkitchen objects were more evenly distributed across (normative) expectancy ratings.

\section{Procedure}

Participant were instructed to categorise their responses for both recall and 3AFC: If they did not remember seeing the object at all, they were told to indicate "no memory". If they remembered the object, but had little idea where it was, they were to indicate "guess". If they did not initially remember where it was, but once they had placed it (in recall) or compared the three choices (in 3AFC), that location just looked familiar, they were to indicate "familiar". Finally, if they immediately remembered where the object was when they saw it (because, for instance, they remembered what they thought when they saw the object), then they were to indicate "remember".

\section{Statistical analysis}

Statistical analysis was identical to Experiment 1, with BFs based on the same zero-centred priors. However, we also used the posterior distributions of Experiment 1 as prior distributions by estimating the family-specific parameters of the Student's t-distribution (see Supplementary Material S5). We used the marginal distribution, ignoring any correlation between parameters, and used the same factor to scale expectancy ratings as in Experiment 1, to ensure comparable expectancy ratings across experiments and hence correct priors (so the SD of these ratings in Experiment 2 was close to, but not identical to, 0.5$)$. The BF from this second model allowed us to update the posterior belief in favour of our hypotheses (what we call " $P B_{10}$ "), given the data from both experiments (calculated by multiplying this $\mathrm{BF}$ with the $\mathrm{BF}$ from the previous experiment).

Because of the lack of effects of including a general expectancy covariate in Experiment 1, we do not report this model here, but we do report in Supplementary Sections S.6.2 - S.6.3 for completeness. 
Remember/familiar judgements were initially analysed in line with pre-registered analysis of the mean expectancy rating for remember and familiar judgments, but further simulation showed that this trialaveraged analysis is biased by boundary effects on expectancy values (see Supplementary Section S.6.2). Therefore, we analysed them using the same single-trial logistic regression model as for overall accuracy. To estimate the probability of recollection, an outcome of 1 was used for "remember" judgments, and an outcome of 0 otherwise. There is debate over the best way to estimate familiarity, i.e., whether familiarity and recollection are redundant, independent or exclusive (Knowlton \& Squire, 1995). To model redundancy, familiarity was estimated with an outcome of 1 for "remember" or "familiar" responses, and 0 otherwise; to model independence, an outcome of 1 was used for "familiar" responses, but only trials that were not given a "remember" response were included; to model exclusivity, familiarity was estimated as for independence, and recollection was estimated by an outcome of 1 for "remember" responses, but only trials that were not given a "familiar" response were included. In the main text, we report results from the most popular independence model (Yonelinas \& Jacoby, 1995), but the results for the redundant and exclusive models are shown in Supplementary Sections S.6.2 - S.6.3, and did not affect the conclusions.

\section{Results}

An average of $2.75(S D=2.55)$ objects were reported as 'not seen' and therefore excluded from further analysis. The distribution of individual expectancy ratings for kitchen and non-kitchen objects was also more evenly spread across expectancy (Supplementary Figure S4).

For recall (Figure $2 \mathrm{C}$ ), there was evidence for a linear term, $\mathrm{BF}_{10}=2.95, \beta=0.35(95 \% \mathrm{Cl}[-0.14,0.85])$, $\mathrm{PB}_{10}=0.64$, and more importantly, continued strong evidence for a positive quadratic term, $\mathrm{BF}_{10}=$ $6.15, \beta=1.85(95 \% \mathrm{Cl}[0.75,2.98]), \mathrm{PB}_{10}=328.78$. 
Like in Experiment 1, there was a bias for incorrect recall to be drawn to expected locations (see Supplementary Section S.6.2). Focusing on the 3AFC therefore (Figure 2D), there was a clear linear term, $\mathrm{BF}_{10}=75.21, \beta=0.71(95 \% \mathrm{Cl}[0.22,1.21]), \mathrm{PB}_{10}=12.87$, and more importantly, a clear quadratic term, $\mathrm{BF}_{10}=12.11, \beta=1.31(95 \% \mathrm{Cl}[0.31,2.34]), \mathrm{PB}_{10}=25.15$. The positive linear term produced an asymmetry in the U-shape towards expected locations.

Assuming that recollection and familiarity are independent, the estimate of recollection from $3 \mathrm{AFC}$ responses (Figure $3 \mathrm{~A}$ ) showed no evidence for a linear term, $\mathrm{BF}_{10}=0.43, \beta=0.274(95 \% \mathrm{Cl}[-0.235$, 0.802]), but strong evidence for a quadratic term, $\mathrm{BF}_{10}=65.30, \beta=1.765(95 \% \mathrm{Cl}[0.636,2.977])$. $\mathrm{By}$ comparison, the estimate of familiarity (Figure $3 \mathrm{~B}$ ) showed no evidence for a linear term, $\mathrm{BF}_{10}=0.41$, $\beta=0.246(95 \% \mathrm{Cl}[-0.379,0.892])$, or quadratic term, $\mathrm{BF}_{10}=0.62, \beta=-0.104(95 \% \mathrm{Cl}[-1.37,1.168])$.
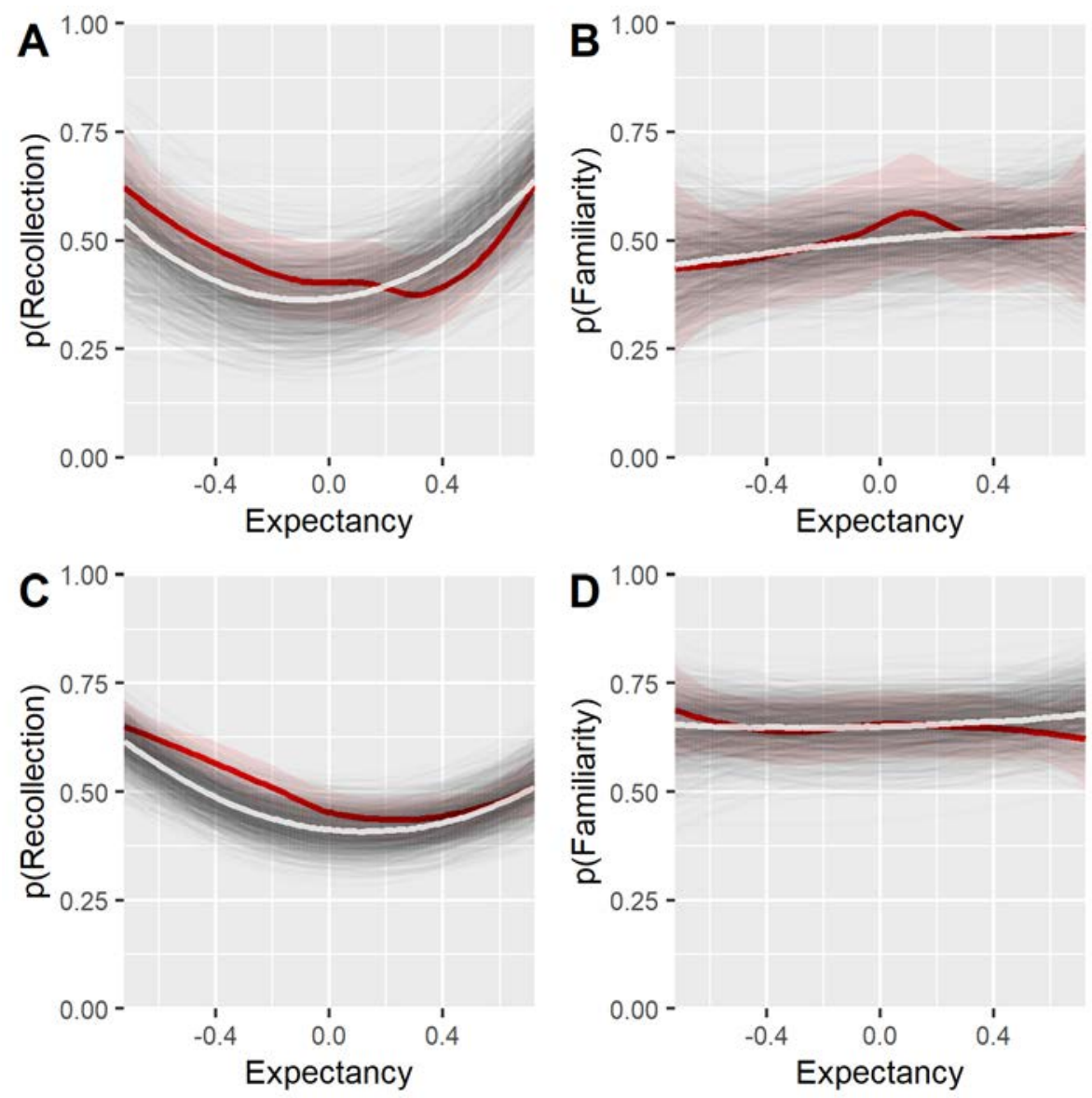
Figure 3. $3 A F C$ recognition performance based on recollection (left panel) and familiarity (right panel) estimates across Experiment 2 and 3 (rows). The red line shows locally weighted smoothing (loess) of data to illustrate how well the average model (the white line) represents the data. The thin grey lines show 1,000 randomly selected fits from the posterior distributions, to illustrate the uncertainty of the average model. Expectancy originally ranged from -100 to +100 , but were scaled to have a SD=0.5 in order to enable standard priors.

\section{Discussion}

Experiment 2 replicated the $\mathrm{U}$-shape in recall of Experiment 1 (and using a more even distribution of kitchen/non-kitchen objects across expectancy values), but now also found a U-shape in 3AFC, which rules out a contribution from guessing expected locations. This confirmed the first of our original hypotheses. However, the results did not support our second hypothesis, that the advantage for unexpected locations would be accompanied by recollection, while that for expected locations would be accompanied by familiarity. Rather, we found that both ends of the U-shape were accompanied by recollection, whereas familiarity showed little effect of expectancy.

One limitation of Experiments 1-2 is that the same set of 20 object-location pairings were used for all participants (even if they were changed slightly across experiments), raising the possibility that the Ushape was a quirk of these particular pairings. Therefore, in Experiment 3, we created five new sets of object-location pairings, counterbalanced across participants, to check the U-shape generalised.

\section{Experiment 3}

Experiment 3 was run to check we could again replicate the U-shape, particularly in $3 A F C$, but this time across a large range of object-location pairings.

\section{Methods}

\section{Participants}

A total of 75 participants ( 48 females, 26 male and 1 non-binary, mean age 25.29 years, SD $=5.11$ years) were tested, counterbalanced across the 5 stimulus sets. All participants were run on exactly 
the same paradigm, but a subset of 51 ( 37 females, 13 male and 1 non-binary, mean age 25.45 years, $S D=5.68$ years) had previously also made judgments about a list of 288 words, as part of a separate Registered Report looking at the effect of the subsequent iVR experience on incidental memory for those words (Quent \& Henson, 2020). The words were not related to kitchens or objects used in the iVR phase, and participants were told the words were not relevant to the iVR phase. These two experiments were pre-registered at (https://osf.io/kcr2q \& https://osf.io/b9dqg).

\section{Materials, Procedure \& Statistic analysis}

These were identical to Experiment 2, except that five new sets of 20 object-location pairings were selected from our normative data, each chosen to maximise the range of expectancy values (materials available here https://osf.io/y72ew/ \& https://osf.io/4sw2t/).

\section{Results}

An average of $2.13(\mathrm{SD}=1.61$ ) objects were reported as 'not seen' and excluded from further analysis.

For recall (Figure 2E), the results showed evidence against a linear term, $\mathrm{BF}_{10}=0.18, \beta=0.01(95 \% \mathrm{Cl}$ $[-0.22,0.24]), \mathrm{PB}_{10}=0.12$, but evidence for a quadratic term, $\mathrm{BF}_{10}=5.52, \beta=1.08(95 \% \mathrm{Cl}[0.48,1.66])$, $\mathrm{PB}_{10}=522.75$. Like in Experiments 1-2, there was a bias for incorrect recalls to be drawn towards expected locations (Supplementary Section S.6.3).

For 3AFC (Figure 2F), there was little evidence for a linear term, $\mathrm{BF}_{10}=0.14, \beta=0.22(95 \% \mathrm{Cl}[-0.04$, 0.49]), $\mathrm{PB}_{10}=0.64$, but evidence for a quadratic term, $\mathrm{BF}_{10}=2.71, \beta=0.96$ (95 \% Cl [0.34, 1.57]), particularly in terms of the posterior belief, $\mathrm{PB}_{10}=65.89$.

For recollection estimates from $3 \mathrm{AFC}$ (Figure $3 \mathrm{C}$ ), there was evidence for a linear term, $\mathrm{BF}_{10}=14.26$, $\beta=-0.271(95 \% \mathrm{Cl}[-0.508,-0.035])$, but not when combined with previous experiments, $\mathrm{PB}_{10}=1.44$, but more importantly, strong evidence for a quadratic term, BF10 $=14.86, \beta=1.23(95 \% \mathrm{Cl}[0.64$, 1.82]), $\mathrm{PB}_{10}=1088.55$. For familiarity (Figure $3 \mathrm{D}$ ), there was little evidence for a linear, $\mathrm{BF}_{10}=0.24, \beta$ $=0.08(95 \% \mathrm{Cl}[-0.309,0.465]), \mathrm{PB}_{10}=0.20$ or quadratic, $\mathrm{BF}_{10}=0.52, \beta=0.03(95 \% \mathrm{Cl}[-0.84,0.90])$, 
$\mathrm{PB}_{10}=0.43$, term. See Supplementary Section S.6.3 for results under different assumptions about relationship between recollection and familiarity.

Finding evidence for a quadratic term in the absence of a linear effect supports a U-shaped function, but a stronger test is to check that both ends of a U-shape are reliable, by testing for opposite linear slopes; so-called "interrupted regression" (e.g., Simonsohn, 2018). As pre-registered (see https://osf.io/b9dqg), we tested this by combining data across all experiments. To determine slopes of interrupted linear regressions, one must find a "breaking-point" somewhere along the continuum, at which the two slopes meet. We did this by testing 10 equally-spaced breaking points within the middle $80 \%$ range of the expectancy ratings. Simulations showed that the false positive rate remained under $5 \%$ for testing these 10 breaking points (see https://jaquent.github.io/post/finding-a-u-shapewith-bayesian-interrupted-regression/).

For recall, the strongest effect was with a breakpoint of +0.05 , where the estimated leftward slope (towards negative expectancy values) was $\beta=-0.75$ (95 $\% \mathrm{Cl}[-1.31,-0.19]), \mathrm{BF}_{10}=22.09$, and the rightward slope was $\beta=+0.84(95 \% \mathrm{Cl}[0.17,1.51]), \mathrm{BF}_{10}=14.56$. For $3 \mathrm{AFC}$, the breakpoint of -0.26 had a leftward slope of $\beta=-1.17$ (95\% Cl $[-2.2,-0.1]), \mathrm{BF}_{10}=10.68$, and a rightward slope of $\beta=+0.55$ (95\% Cl $[0.11,0.99]), \mathrm{BF}_{10}=9.03$. For recollection estimates of $3 \mathrm{AFC}$, the breakpoint of +0.05 had a leftward slope of $\beta=-1.09$ (95 \% Cl $[-1.66,-0.53]), \mathrm{BF}_{10}=394.65$, and rightward slope of $\beta=+1.01$ (95 \% Cl $[0.34,1.72]), \mathrm{BF}_{10}=65.20$. These results provide strong support for both sides of the U-shape being reliable in both measures of memory, and for recollection in particular. Full results, including for familiarity, can be found in Supplementary Section S.7.

\section{Discussion}

Experiment 3 confirmed the U-shape function for both recall and recognition as a function of objectlocation expectancy, particularly when combined with Experiments 1 and 2, and confirmed that both ends of this U-shape were associated with recollection. This was apparent by strong evidence for a positive quadratic component in the absence of a linear component, and in more strict interrupted 
regression analyses, which confirmed that both sides of the U-shape showed a significant linear effect of opposite sign.

\section{General Discussion}

We were able to confirm our first prediction that memory can be a U-shaped function of the expectancy of an occurrence, with better memory for highly expected or highly unexpected information. This prediction was derived from a model called SLIMM that presumes that different brain systems underlie the two ends of the expectancy dimension, and reconciles many previous studies that have claimed superior memory for either expected (schema-congruent) information or unexpected (surprising) events: whether one finds one advantage or the other depends on the relative position of the two experimental conditions along the expectancy continuum. Only by exploring a continuous and extreme range of expectancy can one see the larger picture. The present finding replicates and extends our previous demonstration of a U-shape using 3 experimental conditions (Greve et al., 2019), and importantly demonstrates that the U-shape also occurs using rich, preexperimental knowledge, rather than a simple rule learned during an experiment. Indeed, our use of iVR allowed us to test memory quickly (after less than a minute of encoding) in a realistic situation, while simultaneously providing the precise experimental control needed to measure memory.

In particular, our results may reconcile those from previous studies that have examined memory for objects within familiar types of rooms. In their seminal work, Brewer and Treyens (1981) reported that memory was positively correlated with schema expectancy. However, the opposite effect, where atypical objects in a room were remembered better, has also been reported (Lampinen et al., 2001; Pezdek et al., 1989; Prull, 2015). The discrepancy between these studies most likely reflects two factors: 1) with only restricted range of expectancy, the relative level of memory depends on the position of those points on the U-shaped continuum (as noted above), and 2) the precise type of memory assessed. Both of these issues apply, for example, to the study of Lew and Howe (2017), 
which is most similar to the present study, except it used realistic photos of familiar room-types ${ }^{1}$. Like here, the photos showed room-congruent objects at expected and unexpected locations, as well as room-incongruent objects. At test, objects either stayed in same location or shifted to a different location. Recognition of objects was better for room-incongruent objects as well as room-congruent objects at unexpected locations, relative to room-congruent objects at expected locations. However, their recognition test was likely also influenced by memory for the object themselves, whether or not their location was switched (whereas here, we ignored the rare trials when an object was not recognised at all, and our "recognition" refers to recognition of the object's location). The predictions of SLIMM tested here relate to associative memory, i.e., between an object and its location, rather than item memory (Cohen et al., 1997; Mayes et al., 2004). Indeed, when Lew and Howe (2017) tested memory by recall instead, memory for room-congruent objects at unexpected locations was impaired relative to room-congruent objects at expected locations. The authors explained this in terms of schemas acting differently on item and associative (location) memory, whereby an unexpected location attracts attention, but also activates schema-congruent bindings that interfere with memory (see also Bower et al., 1979). However, an alternative explanation for their dissociation between recognition and recall is that their recall test, but not recognition test, was influenced by guessing of room-congruent locations when memory failed (e.g., Bayen et al., 2000). This is indeed what we found when examining errors in our recall test. By controlling for this guessing bias in our 3AFC, through matching the expectancy of the foils, we were able to reveal an additional advantage of memory for unexpected locations. Thus, it appears critical to not only explore a range of expectancy values, but

1 The only previous study to investigate memory for objects using iVR, of which we are aware, was by Draschkow \& Võ (2017). These authors examined how room schemas influence interactions with, and memory for, objects. Participants were asked to either arrange objects in a virtual room in a manner that was consistent or inconsistent with their expected arrangement. Participants spent more time handling and searching for inconsistently arranged objects, and consistently arranged objects were recalled more accurately than inconsistently arranged objects (possibly reflecting the guessing bias described above). While this study is a clever illustration of the use of iVR to study the effect of schema, it did not explicitly examine the continuous relationship between expectancy and memory. 
also control for other ways in which schema can influence memory performance, such as at retrieval as well as encoding.

However, we failed to confirm the second prediction of the SLIMM model, namely that one side of the U-shape (high unexpectancy) would be associated with recollection of contextual details while the other side (high expectancy) would be associated only with a feeling of familiarity (also see Kafkas \& Montaldi, 2018). Indeed, our Bayesian analysis provided evidence against any effect of expectancy for familiarity, and evidence instead for a U-shaped function of recollection. One possibility is that the SLIMM conception of the relationship between expectancy/schema-congruency and recollection versus familiarity is incorrect. Another possibility is that our assessment of memory for the location of objects (rather than the objects themselves; see above) inherently requires the same associative mechanisms that support recollection (and which are assumed to be supported by the hippocampus within the MTL system). One way to test the latter would be to examine memory for the objects themselves (such as their perceptual details), rather than their location, and see if such "item memory" is associated with recollection when the object was highly unexpected, but familiarity when the object was highly expected.

Our study raises interesting future questions, though also possible limitations. One interesting question is whether the U-shape applies to all types of expectancy, or only predictions deriving from pre-existing knowledge, i.e. schema. For example, would the same U-shape emerge for events that are expected or unexpected given an episodic context, such as a temporal sequence of items that enable a prediction for the next item (such as the isolation effect, von Restorff, 1933). Another interesting question is whether the form of the U-shape varies across development and/or ageing. For example, one might expect the advantage for schema-congruent events to increase during childhood, and the advantage for schema-incongruent events, if they rely more on episodic memory, to decline in old age. One limitation of the present findings is whether they generalise to other types of memory beyond object locations. While we have shown a U-shape for verbal rules (Greve et al., 2019), the same may not apply to other stimuli and other types of memory test. A second limitation of the 
present study is that we only tested immediate memory, and it is possible that the U-shape changes as the retention interval increases, e.g., following consolidation processes that might occur overnight. Either way, our results demonstrate the important role of prior knowledge in shaping the encoding of new memories, and unify two factors (schema-congruency versus surprise) that have previously tended to be studied separately.

\section{References}

Alba, J. W., \& Hasher, L. (1983). Is memory schematic? Psychological Bulletin, 93(2), 203-231. https://doi.org/10.1037/0033-2909.93.2.203

Anderson, J. R. (1981). Effects of prior knowledge on memory for new information. Memory \& Cognition, 9(3), 237-246. https://doi.org/10.3758/BF03196958

Bartlett, F. C. (1932). Remembering: A Study in Experimental and Social Psychology. Cambridge University Press.

Bayen, U. J., Nakamura, G. V., Dupuis, S. E., \& Yang, C.-L. (2000). The use of schematic knowledge about sources in source monitoring. Memory \& Cognition, 28(3), 480-500. https://doi.org/10.3758/BF03198562

Bein, O., Livneh, N., Reggev, N., Gilead, M., Goshen-Gottstein, Y., \& Maril, A. (2015). Delineating the Effect of Semantic Congruency on Episodic Memory: The Role of Integration and Relatedness. PLOS ONE, 10(2), e0115624. https://doi.org/10.1371/journal.pone.0115624

Bower, G. H., Black, J. B., \& Turner, T. J. (1979). Scripts in memory for text. Cognitive Psychology, 11(2), 177-220. https://doi.org/10.1016/0010-0285(79)90009-4

Bransford, J. D., \& Johnson, M. K. (1972). Contextual prerequisites for understanding: Some investigations of comprehension and recall. Journal of Verbal Learning and Verbal Behavior, 11(6), 717-726. https://doi.org/10.1016/S0022-5371(72)80006-9

Brewer, W. F., \& Treyens, J. C. (1981). Role of schemata in memory for places. Cognitive Psychology, 13(2), 207-230. https://doi.org/10.1016/0010-0285(81)90008-6

Brod, G., Hasselhorn, M., \& Bunge, S. A. (2018). When generating a prediction boosts learning: The element of surprise. Learning and Instruction, 55, 22-31. https://doi.org/10.1016/j.learninstruc.2018.01.013

Brod, G., \& Shing, Y. L. (2019). A boon and a bane: Comparing the effects of prior knowledge on memory across the lifespan. Developmental Psychology, 55(6), 1326-1337. https://doi.org/10.1037/dev0000712

Bürkner, P.-C. (2017). brms: An R Package for Bayesian Multilevel Models Using Stan. Journal of Statistical Software; Vol 1, Issue 1 (2017). https://www.jstatsoft.org/v080/i01 
Bürkner, P.-C. (2018). Advanced Bayesian Multilevel Modeling with the R Package brms. The R Journal, 10(1), 395. https://doi.org/10.32614/RJ-2018-017

Carpenter, B., Gelman, A., Hoffman, M. D., Lee, D., Goodrich, B., Betancourt, M., Brubaker, M., Guo, J., Li, P., \& Riddell, A. (2017). Stan: A Probabilistic Programming Language. Journal of Statistical Software; Vol 1, Issue 1 (2017). https://www.jstatsoft.org/v076/i01

Cohen, N. J., Poldrack, R. A., \& Eichenbaum, H. (1997). Memory for Items and Memory for Relations in the Procedural/Declarative Memory Framework. Memory, 5(1-2), 131-178. https://doi.org/10.1080/741941149

Craik, F. I. M., \& Tulving, E. (1975). Depth of processing and the retention of words in episodic memory. Journal of Experimental Psychology: General, 104(3), 268-294. https://doi.org/10.1037/00963445.104.3.268

Draschkow, D., \& Võ, M. L.-H. (2017). Scene grammar shapes the way we interact with objects, strengthens memories, and speeds search. Scientific Reports, 7(1), 16471. https://doi.org/10.1038/s41598-017-16739-x

Gardiner, J. M., Ramponi, C., \& Richardson-Klavehn, A. (2002). Recognition memory and decision processes: A meta-analysis of remember, know, and guess responses. Memory (Hove, England), 10(2), 83-98. https://doi.org/10.1080/09658210143000281

Gelman, A., Jakulin, A., Pittau, M. G., \& Su, Y.-S. (2008). A weakly informative default prior distribution for logistic and other regression models. The Annals of Applied Statistics, 2(4), 1360-1383. https://doi.org/10.1214/08-AOAS191

Greve, A., Cooper, E., Kaula, A., Anderson, M. C., \& Henson, R. (2017). Does prediction error drive oneshot declarative learning? Journal of Memory and Language, 94, 149-165. https://doi.org/10.1016/j.jml.2016.11.001

Greve, A., Cooper, E., Tibon, R., \& Henson, R. N. (2019). Knowledge is power: Prior knowledge aids memory for both congruent and incongruent events, but in different ways. Journal of Experimental Psychology: General, 148(2), 325-341. https://doi.org/10.1037/xge0000498

Gronau, Q. F., Erp, S. V., Heck, D. W., Cesario, J., Jonas, K. J., \& Wagenmakers, E.-J. (2017). A Bayesian model-averaged meta-analysis of the power pose effect with informed and default priors: The case of felt power. Comprehensive Results in Social Psychology, 2(1), 123-138. https://doi.org/10.1080/23743603.2017.1326760

Hunt, R. R., \& Worthen, J. B. (Eds.). (2006). Distinctiveness and Memory. Oxford University Press. https://doi.org/10.1093/acprof:oso/9780195169669.001.0001

Kafkas, A., \& Montaldi, D. (2018). How do memory systems detect and respond to novelty? Neuroscience Letters, 680(July 2017), 60-68. https://doi.org/10.1016/j.neulet.2018.01.053

Knowlton, B. J., \& Squire, L. R. (1995). Remembering and knowing: Two different expressions of declarative memory. Journal of Experimental Psychology: Learning, Memory, and Cognition, 21(3), 699-710. https://doi.org/10.1037/0278-7393.21.3.699

Lampinen, J. M., Copeland, S. M., \& Neuschatz, J. S. (2001). Recollections of things schematic: Room schemas revisited. Journal of Experimental Psychology: Learning, Memory, and Cognition, 27(5), 1211-1222. https://doi.org/10.1037/0278-7393.27.5.1211 
Lew, A. R., \& Howe, M. L. (2017). Out of place, out of mind: Schema-driven false memory effects for object-location bindings. Journal of Experimental Psychology: Learning Memory and Cognition, 43(3), 404-421. https://doi.org/10.1037/xIm0000317

Mayes, A. R., Holdstock, J. S., Isaac, C. L., Montaldi, D., Grigor, J., Gummer, A., Cariga, P., Downes, J. J., Tsivilis, D., Gaffan, D., Gong, Q., \& Norman, K. A. (2004). Associative Recognition in a Patient With Selective Hippocampal Lesions and Relatively Normal Item Recognition. Hippocampus, 14(6), 763784. https://doi.org/10.1002/hipo.10211

Morey, R. D., \& Rouder, J. N. (2018). BayesFactor: Computation of Bayes Factors for Common Designs. https://cran.r-project.org/package=BayesFactor

Pezdek, K., Whetstone, T., Reynolds, K., Askari, N., \& Dougherty, T. (1989). Memory for real-world scenes: The role of consistency with schema expectation. Journal of Experimental Psychology: Learning, Memory, and Cognition, 15(4), 587-595. https://doi.org/10.1037/0278-7393.15.4.587

Prull, M. W. (2015). Adult age differences in memory for schema-consistent and schema-inconsistent objects in a real-world setting. Aging, Neuropsychology, and Cognition, 22(6), 731-754. https://doi.org/10.1080/13825585.2015.1037821

Quent, J. A., \& Henson, R. (2020). The effect of a novel immersive virtual reality experience on memory for unrelated information: Modulations by encoding task and retrieval quality. OSF. https://osf.io/y72ew/

R Core Team. (2019). R: A language and environment for statistical computing. Vienna, Austria.

Rajaram, S. (1993). Remembering and knowing: Two means of access to the personal past. Memory \& Cognition, 21(1), 89-102. https://doi.org/10.3758/BF03211168

Simonsohn, U. (2018). Two Lines: A Valid Alternative to the Invalid Testing of U-Shaped Relationships With Quadratic Regressions. Advances in Methods and Practices in Psychological Science, 1(4), 538555. https://doi.org/10.1177/2515245918805755

Tulving, E. (1985). Memory and consciousness. Canadian Psychology/Psychologie Canadienne, 26(1), 1-12. https://doi.org/10.1037/h0080017

van Buuren, M., Kroes, M. C. W., Wagner, I. C., Genzel, L., Morris, R. G. M., \& Fernández, G. (2014). Initial Investigation of the Effects of an Experimentally Learned Schema on Spatial Associative Memory in Humans. Journal of Neuroscience, 34(50), 16662-16670. https://doi.org/10.1523/JNEUROSCI.236514.2014

van Kesteren, M. T. R., Ruiter, D. J., Fernández, G., \& Henson, R. N. (2012). How schema and novelty augment memory formation. Trends in Neurosciences, 35(4), 211-219. https://doi.org/10.1016/j.tins.2012.02.001

von Restorff, H. (1933). Über die Wirkung von Bereichsbildungen im Spurenfeld. Psychologische Forschung, 18(1), 299-342. https://doi.org/10.1007/BF02409636

Wagenmakers, E.-J., Lodewyckx, T., Kuriyal, H., \& Grasman, R. (2010). Bayesian hypothesis testing for psychologists: A tutorial on the Savage-Dickey method. Cognitive Psychology, 60(3), 158-189. https://doi.org/10.1016/j.cogpsych.2009.12.001 
Yonelinas, A. P., \& Jacoby, L. L. (1995). The relation between remembering and knowing as bases for recognition: Effects of size congruency. Journal of Memory and Language, 34(5), 622-643. https://doi.org/10.1006/jmla.1995.1028 